\title{
Prospects for Semileptonic B decays at Belle II
}

\section{Guglielmo De Nardo* on behalf of the Belle II Collaboration}

Universitá di Napoli Federico II and INFN Sezione di Napoli

E-mail: denardo@na.infn.it

The Belle II experiment is a substantial upgrade of the Belle detector and will operate at the SuperKEKB energy-asymmetric $e^{+} e^{-}$collider. The design luminosity of SuperKEKB is $8 \times$ $10^{35} \mathrm{~cm}^{-2} \mathrm{~s}^{-1}$ and the Belle II experiment aims to record $50 \mathrm{ab}^{-1}$ of data, a factor of 50 more than the Belle experiment. With this amount of data, decays sensitive to physics beyond the Standard Model can be studied with unprecedented precision. In this contribution we will present our prospects for studying lepton flavor non-universality with the modes $B \rightarrow D^{(*)} \tau \nu$ and for the measurement of the CKM parameter $\left|V_{u b}\right|$.

An Alpine LHC Physics Summit (ALPS2018)

15-20 April, 2018

Obergurgl, Austria

${ }^{*}$ Speaker. 


\section{Introduction}

The B-Factory experiments BaBar[1] and Belle[2] have performed a large number of measurements of unprecedented precision in the sector of Heavy Flavour Physics providing a stringent test of the Standard Model (SM) of weak interactions[3]. Despite the success of the SM in describing the observed phenomenology, some measurements showed signs of remarkable tension, at the level of several standard deviations, with respect to theoretically predictions. The most significant example is the measurement of the ratio $R\left(D^{(*)}\right)$ between the semileptonic B decays $B \rightarrow D^{(*)} \tau \nu$ and $B \rightarrow D^{(*)} l v$, where $l$ are the lighter leptons, pointing to a violation of the lepton flavour universality implied by the SM. Measurements from BaBar, Belle and LHCb show a combined $4 \sigma$ excess of signal with respect to the SM prediction. Another intriguing tension has also been observed in the determination of $\left|V_{u b}\right|$ between exclusive charmless semileptonic decays and inclusive ones. In order to improve the sensitivity to Physics more fundamental than the SM, KEKB collider has been upgraded to reach an instantaneous luminosity 40 times higher, in order to collect an experimental data sample 50 times larger than the one accumulated by the Belle Experiment. The Belle detector has been upgraded to Belle II[4] as well, to cope with the higher rate of events and the higher backgrounds produced by the accelerator.

\section{Experimental techniques}

The reconstruction of semileptonic decays in electron and muon modes is experimentally complicated by the presence of a neutrino in the final state that escapes direct detection. The experimental signature is an unbalance of the total momentum and reconstructed energy, that can be mimicked by physics backgrounds with undetected particles going out of acceptance or neutral hadrons, since they are not vetoed with $100 \%$ efficiency. In the case of a $\tau$ lepton, the situation is even worse because one or two additional neutrinos are produced in its subsequent decay.

Nevertheless, the unique experimental conditions at the $e^{+} e^{-}$B factories, where exactly one pair of B mesons is produced, and the fact the detector encloses the interaction region almost hermetically, permits to estimate the four-momentum of the undetected neutrinos from the mesurements of the momentum and energy of all the other particles reconstructed in the event. This procedure is commonly denoted as an untagged mesurement. In addition to the signal $B$ decaying semileptonically $\left(B_{\text {sig }}\right)$, also the other B in the event can be explicitly reconstructed in a variety of exclusive modes $\left(B_{t a g}\right)$, so that the entire event is reconstructed. At the cost of a reduced reconstruction efficiency, strong constraints can be put imposing balance of the reconstructed four-momentum and on the energy deposited in the electromagnetic calorimeter.

The related most discriminating observables that have been commonly used are the missing mass $M_{\text {miss }}$ and the residual energy deposited in the calorimeter $E_{\text {extra }}$. The missing mass squared is defined as $M_{\text {miss }}^{2}=\left(p_{e^{+} e^{-}}-p_{B_{s i g}}-p_{B_{t a g}}\right)^{2}$, where $p_{e^{+} e^{-}}$is the known 4-momentum of the colliding beams, and the $p_{B_{s i g}}$ and $p_{B_{t a g}}$ are the measured 4-momenta of the reconstructed signal and tag $B$ meson, respectively. In the case of the charged lepton being an electron or a muon, only one neutrino is missing and $M_{\text {miss }}^{2}$ peaks at zero. In the case of semi-tauonic decays, more neutrinos escape detection and the signal populates the high mass tail of the $M_{m i s s}^{2}$. Extra energy in the calorimeter, $E_{\text {extra }}$, is defined as the sum of the energy deposits in the calorimeter that have not 


\begin{tabular}{ccc}
\hline & $5 \mathrm{ab}^{-1}$ & $50 \mathrm{ab}^{-1}$ \\
\hline$R_{D}$ & $( \pm 6.0 \pm 3.9) \%$ & $( \pm 2.0 \pm 2.5) \%$ \\
$R_{D^{*}}$ & $( \pm 3.0 \pm 2.5) \%$ & $( \pm 1.0 \pm 2.0) \%$ \\
$P_{\tau}\left(D^{*}\right)$ & $\pm 0.18 \pm 0.08$ & $\pm 0.06 \pm 0.04$ \\
\hline
\end{tabular}

Table 1: Expected precision for $R_{D^{(*)}}$ and $P_{\tau}\left(D^{*}\right)$ at Belle II, given as the relative uncertainty for $R_{D^{(*)}}$ and absolute for $P_{\tau}\left(D^{*}\right)$. The values given are the statistical and systematic errors respectively.

been associated with the reconstructed decay products of the $B_{t a g}$ or the $B_{\text {sig }}$. For signal events, where the full event is exclusively reconstructed, $E_{\text {extra }}$ is expected to be zero or very small because of contributions from beam background hits and split-offs from imperfect reconstruction of either the tag or the signal side. On the contrary, most of the background events are distributed toward higher $E_{\text {extra }}$ due to the contribution from additional clusters, produced by unassigned tracks and neutrals from the mis-reconstructed tag and/or signal $B$ mesons. Past measurements from BaBar and Belle have been performed using three different experimental techniques, differing in the way the $\operatorname{tag} B$ is reconstructed. In untagged analyses, the missing energy and momentum of the whole event are used to determine the 4-momentum of the missing neutrino from the signal semileptonic decay. Measurements where the $\operatorname{tag} B$ meson is reconstructed in a large variety of decays modes are commonly denoted as tagged measurements. Semileptonic tagging involves partial reconstruction of a $B_{t a g} \rightarrow D^{(*)} \ell v_{\ell}$ decay as the tagging mode. In this case, two neutrinos are present in the event and the 4-momentum of the $B_{\text {sig }}$ cannot be fully constrained. In full reconstruction tagging, a hadronically decaying $B_{\text {tag }}$ meson is reconstructed. In Belle II, the reconstruction of the tag $B$ has been further improved for an higher reconstruction efficiency with same level of purity using machine learning techniques[5].

\section{Semitauonic decays}

The decay $B \rightarrow D^{(*)} \tau \nu$ is described in the SM with $b \rightarrow c \tau \nu$ tree-level transitions mediated by a virtual $W$ boson, exactly like in the electron and muon case. Therefore, the ratios of the tauonic branching fraction with respect to the lighter leptons

$$
R_{D^{(*)}}=\frac{\operatorname{Br}\left(B \rightarrow D^{(*)} \tau \nu_{\tau}\right)}{\operatorname{Br}\left(B \rightarrow D^{(*)} \ell v_{\ell}\right)},
$$

are observables independent from $\left|V_{c b}\right|$ and sensitive to new physics (NP) contributions that violate the Lepton Flavour Universality implied by the SM, since theoretical uncertainties in form factors largely cancel out. These ratios have been measured by BaBar [6][7], Belle [8][9] and LHCb [10]. The combination of the measurements is in excess with respect to the SM expectation at the level of $4 \sigma$.

Among the various Belle and BaBar analyses the ones in which the $\tau$ is identified by its leptonic decay modes provide the smallest uncertainties because they have better background rejection power. The most dangerous background originate from $B \rightarrow D^{* *} \ell v_{\ell}$, where $D^{* *}$ mesons are excited charmed mesons higher than the $D^{*}(2010)$ that can be mis-reconstructed as signal once the pions (mainly $\pi^{0}$ 's) from $D^{* *}$ decay are missed. The correct estimation of the $B \rightarrow D^{* *} \ell v_{\ell}$ background yield and minimisation of its systematic uncertainty is a critical aspect. Therefore, it is of 
paramount importance at Belle II to perform the support measurements of $B \rightarrow D^{* *} \ell v_{\ell}$ branching ratios to control the systematic effect of those background processes.

In addition to branching fractions ratios $R\left(D^{(*)}\right)$, the polarisations of the $\tau$ lepton and the $D^{*}$ meson and the $q^{2}$ distribution are also sensitive to NP contributions and help to discriminate among NP models.

The polarizations are defined by

$$
\begin{aligned}
P_{\tau}\left(D^{(*)}\right) & =\frac{\Gamma^{+}-\Gamma^{-}}{\Gamma^{+}+\Gamma^{-}}, \\
P_{D^{*}} & =\frac{\Gamma_{\mathrm{L}}}{\Gamma_{\mathrm{L}}+\Gamma_{\mathrm{T}}},
\end{aligned}
$$

where $\Gamma^{+(-)}$and $\Gamma_{\mathrm{L}(\mathrm{T})}$ are the decay rate with the $\tau$ helicity $+1 / 2(-1 / 2)$ and that with the longitudinally (transversely) polarised $D^{*}$, respectively. Belle II should also be able to perform precise differential measurements in $q^{2}$, and is expected to have some sensitivity to measure the decay angles $\left(\theta_{\ell}, \theta_{V}, \chi\right)$ as it was already performed for $B \rightarrow D^{(*)} \ell v$ decays at Belle and BaBar.

The hadronic $\tau$ decay modes $\tau^{-} \rightarrow h^{-} v_{\tau}$, with $h^{-}=\pi^{-}, \rho^{-}$have been used in a recent Belle analysis of $B \rightarrow D^{*} \tau \nu_{\tau}$ [11] to add a statistically independent measurement of $R\left(D^{*}\right)$ and to measure the polarization $P_{\tau}\left(D^{*}\right)$, exploiting the kinematics of two-body $\tau$ decays. The signal is fitted following the same approach of the hadronic-tag analysis with $\tau^{-} \rightarrow \ell^{-} \bar{v}_{\ell} \nu_{\tau}$, using $M_{\text {miss }}^{2}$ and $E_{\text {extra }}$ to extract the $B \rightarrow D^{*} \ell v_{\ell}$ and the $B \rightarrow D^{*} \tau v_{\tau}$ yields. However, in this case, the most important background originates from hadronic decays $B \rightarrow D^{*} \pi^{+} X$, where $X$ consists of one or more unreconstructed $\pi^{0}, \eta, \gamma$ or pairs of charged particles. Since uncertainties in branching fractions of the exclusive hadronic $B$ decay modes introduce sizeable systematic uncertainty, an extensive set of measurements at Belle II of those modes is highly desirable.

Table 1 shows projections of the precision on $R_{D^{(*)}}$ and $P_{\tau}\left(D^{*}\right)$ for $5 a b^{-1}$ and the full data set of $50 a b^{-1}$, based on the current results from Belle and the expected statistical and experimental improvements at Belle II. As shown in Figure 1, where the expected precisions at Belle II are compared to the current results and SM expectations, the experimental uncertainty will be at the same level of the present theoretical one. Precise measurements of polarisation $P_{\tau}\left(D^{*}\right)$, and decay differentials of kinematical observables at Belle II will allow to test NP scenarios. Figure 2 demonstrates the statistical precision of the $q^{2}$ measurement with $50 \mathrm{ab}^{-1}$ data based on a toy-MC study with the hadron tag analysis.

\section{Determination of $\left|V_{u b}\right|$}

The aim for Belle II is to reach one percent-level determinations of $\left|V_{u b}\right|$ measuring the branching ratios of several exclusive charmless modes $B \rightarrow h \ell v$, where $h$ is a light hadron, and the inclusive rate to charmless semileptonic final state $B \rightarrow X_{u} \ell v$, where $X_{u}$ is an hadronic system originated from the u quark hadronization.

Among the exclusive modes, the most precise determinations of $\left|V_{u b}\right|$ have been obtained from the (untagged) analysis of the $B \rightarrow \pi \ell v$, by BaBar [12] and Belle [13]. The expected increase of almost two order of magnitude of more data from Belle II will allow precise tests of the latticeQCD predictions for the $q^{2}$ dependence. If the efficiency corrected experimental $q^{2}$ shape will be in 

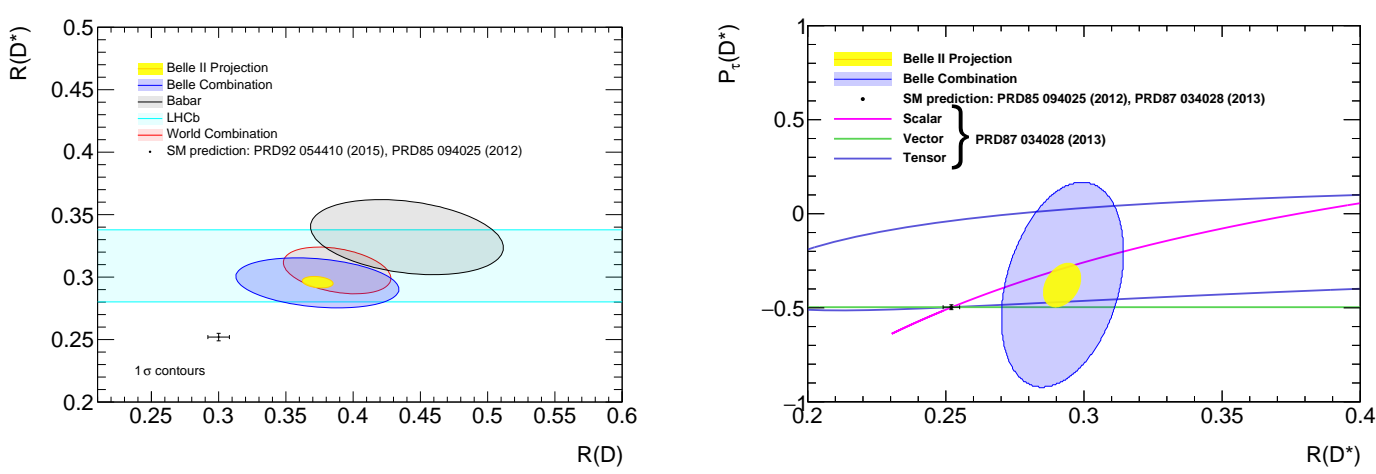

Figure 1: Expected Belle II constraints on the $R_{D^{*}}$ vs $R_{D}$ plane (left) and the $P_{\tau}\left(D^{*}\right)$ vs $R_{D^{*}}$ plane (right) compared to existing experimental constraints from Belle. The SM predictions are indicated by the black points with theoretical error bars. In the right panel, the NP scenarios "Scalar", "Vector" and "Tensor" assume contributions from the operators $\mathscr{O}_{S_{1}}, \mathscr{O}_{V_{1}}$ and $O_{T}$, respectively.
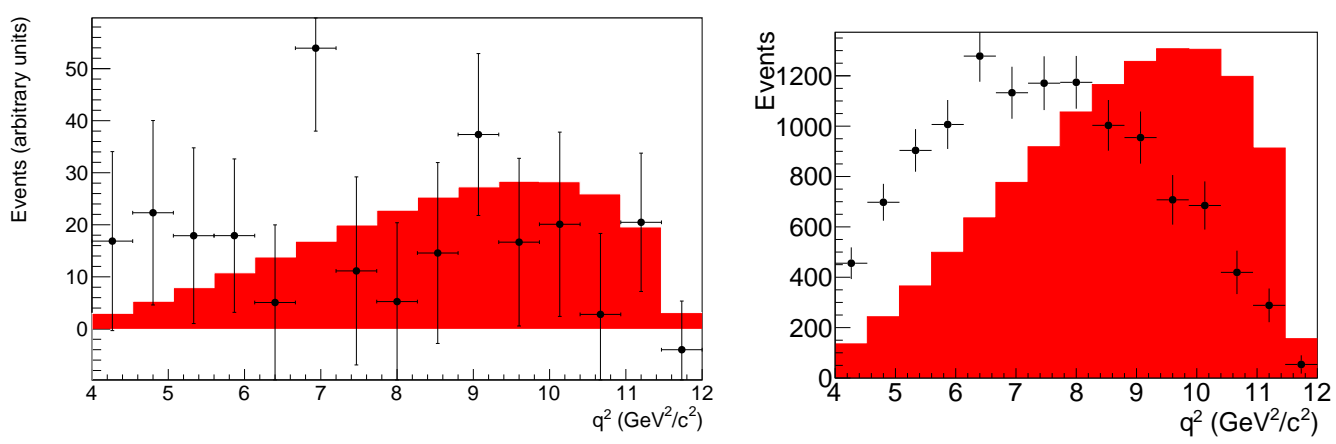

Figure 2: On the left is the $B \rightarrow D \tau \nu q^{2}$ distribution in the hadronic tag analysis and $\tau^{-} \rightarrow \ell^{-} \bar{v}_{\ell} v_{\tau}$ with the full Belle data sample [8]. On the right is the projection to the $50 \mathrm{ab}^{-1}$ of the Belle II data. In both panels, the solid histograms show the predicted distribution shape with the $2 \mathrm{HDM}$ of type II at $\tan \beta / m_{H^{ \pm}}=$ $0.5\left(\mathrm{GeV} / \mathrm{c}^{2}\right)^{-1}$. In the right panel, pseudo-data are shown based on the SM hypothesis.

agreement with lattice QCD predictions, experimental data will be fitted to extract $\left|V_{u b}\right|$. Figure 3 shows the projections to Belle II of $V_{u b}$ uncertainty to various integrated luminosity values. Figure 4 shows the expected $q^{2}$ distribution with $5 a b^{-1}$ of Belle II data.

Measurements of decay rates of exclusive $B \rightarrow h \ell v_{\ell}$ decays, where $h$ denotes a light meson containing a $u$ quark, such as $\pi, \rho, \omega, \eta^{\left({ }^{\prime}\right)}$, etc., and $\ell$ an electron or muon, have been performed in the past using both the tagged and untagged approaches obtaing results of similar precision. The huge increase in statistics of Belle II will allow more detailed studies, including the $q^{2}$ spectrum, helicity angle analysis. Together with improvement of the Lattice-QCD calculations it will contribute to better understanding of the composition of the $b \rightarrow u$ spectrum and, consequently, reducing model dependence in the $\left|V_{u b}\right|$ measurement with inclusive analysis.

The theoretical prediction of the total inclusive semileptonic decay rate to charmless final states is obtained by an operator product expansion. The experimental determination is challenging due to the overwhelming background from charmed semileptonic final states $B \rightarrow X_{c} \ell v$. Suppression of this background requires selecting regions of phase space where the signal is enhanced with respect 


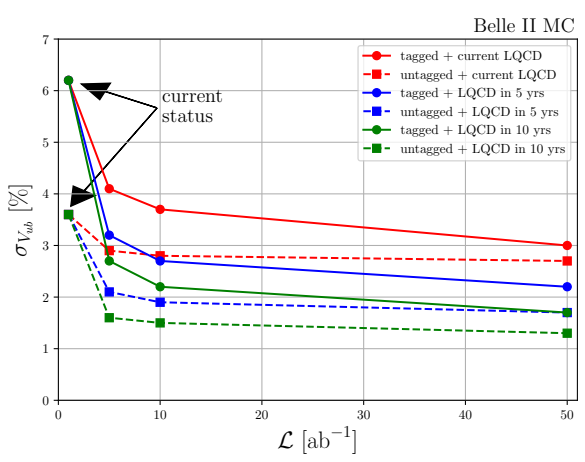

Figure 3: Projections of $V_{u b}$ uncertainty to various luminosity values and lattice-QCD error hypothesis for $B \rightarrow \pi \ell v$ tagged and untagged modes.

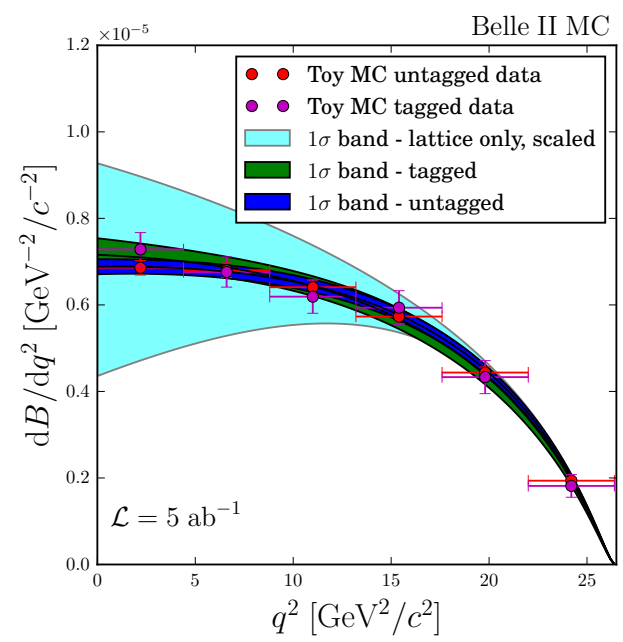

Figure 4: Model independent fits to $q^{2}$ distribution of $B \rightarrow \pi \ell v$ tagged and untagged with $5 a b^{-1}$ data samples, and lattice-QCD error forecasts in 5 years.

to the background, making the extrapolation to the full phase space sensitive to non-perturbative contributions. The most precise measurement from BaBar [14] and Belle [15] uses hadronic tagging and the most inclusive selection requirements. Model dependence is also expected to come from the modelling of the exclusive states composition in $B \rightarrow X_{u} \ell v$ in the efficiency estimation using Monte Carlo simulation. The increase of statistics of Belle II will allow to improve the statistical uncertainty of the measurement and to reduce the systematic uncertainties with supporting measurements able to to constrain the effects described above.

A summary comparing present uncertainties on $\left|V_{u b}\right|$ measurements with projections at Belle II is shown in Table 2 for exclusive (both tagged and untagged) and inclusive analyses.

\section{Conclusions}

Prospects are good for improvements to $\left|V_{u b}\right|$, with both inclusive and exclusive approaches, thanks to more data and better reconstruction performance at Belle II. An high priority goal of Belle 


\begin{tabular}{lccccc}
\hline & Statistical & $\begin{array}{c}\text { Systematic } \\
\text { (reducible, irreducible) }\end{array}$ & Total Exp & Theory & Total \\
\hline$\left|V_{u b}\right|$ exclusive (had. tagged) & & & & & \\
$711 \mathrm{fb}^{-1}$ & 3.0 & $(2.3,1.0)$ & 3.8 & 7.0 & 8.0 \\
$5 \mathrm{ab}^{-1}$ & 1.1 & $(0.9,1.0)$ & 1.8 & 1.7 & 3.2 \\
$50 \mathrm{ab}^{-1}$ & 0.4 & $(0.3,1.0)$ & 1.2 & 0.9 & 1.7 \\
\hline$\left|V_{u b}\right|$ exclusive (untagged) & & & & & \\
$605 \mathrm{fb}^{-1}$ & 1.4 & $(2.1,0.8)$ & 2.7 & 7.0 & 7.5 \\
$5 \mathrm{ab}^{-1}$ & 1.0 & $(0.8,0.8)$ & 1.2 & 1.7 & 2.1 \\
$50 \mathrm{ab}^{-1}$ & 0.3 & $(0.3,0.8)$ & 0.9 & 0.9 & 1.3 \\
\hline$\left|V_{u b}\right|$ inclusive & & & & \\
$605 \mathrm{fb}^{-1}$ (old $B$ tag) & 4.5 & $(3.7,1.6)$ & 6.0 & $2.5-4.5$ & $6.5-7.5$ \\
$5 \mathrm{ab}^{-1}$ & 1.1 & $(1.3,1.6)$ & 2.3 & $2.5-4.5$ & $3.4-5.1$ \\
$50 \mathrm{ab}^{-1}$ & 0.4 & $(0.4,1.6)$ & 1.7 & $2.5-4.5$ & $3.0-4.8$ \\
\hline
\end{tabular}

Table 2: Expected errors in $\left|V_{u b}\right|$ measurements with the Belle full data sample, $5 \mathrm{ab}^{-1}$ and $50 \mathrm{ab}^{-1}$ Belle II data. We use the lattice-QCD projected precision for the future data sets.

II will be to resolve the persistent tension between the exclusive and the inclusive determinations. Current measured anomalies in semi-tauonic modes can be confirmed after only $5 \mathrm{ab}^{-1}$ of data. Critical to this goal is a precise determination from data of the $B \rightarrow D^{* *} \ell v$ background contribution. Differential spectra will be measured with great precision, allowing to distinguish between different NP models.

\section{References}

[1] B. Aubert et al. (BaBar Collaboration), The BABAR Detector, Nucl. Instrum. Meth. A 479, 1 (2002) .

[2] A. Abashian et al. (Belle Collaboration), The Belle Detector, Nucl. Instrum. Meth. A 479, 117 (2002).

[3] A. J. Bevan, B. Golob, et al., The Physics of B Factories, Eur. Phys. J. C 74 (2014) 3026 [arXiv:1406.6311].

[4] T. Abe et al., Belle II Technical Design Report, arXiv:1011.0352.

[5] T. Keck et al., The Full Event Interpretation - An exclusive tagging algorithm for the Belle II experiment, airXiv:1807.08680.

[6] J. P. Lees et al. (BaBar Collaboration), Phys. Rev. Lett. 109, 101802 (2012) [arXiv:1205.5442].

[7] J. P. Lees et al. (BaBar Collaboration), Measurement of an Excess of $B \rightarrow D^{(*)} \tau v$ Decays and Implications for Charged Higgs Bosons, Phys. Rev. D 88, 072012 (2013) [arXiv:1303.0571].

[8] M. Huschle et al. (Belle Collaboration), Measurement of the branching ratio of $B \rightarrow D^{*} \tau v$ relative to $B \rightarrow D^{*} \ell v$ decays with hadronic tagging at Belle, Phys. Rev. D 92, 072014 (2015) [arXiv:1507.03233].

[9] Y.Sato et al. (Belle Collaboration), Measurement of the branching ratio of $B \rightarrow D^{*} \tau v$ relative to $B \rightarrow D^{*} \ell v$ decays with a semileptonic tagging method, Phys. Rev. D 94, 072007 (2016) [arXiv:1607.07923]. 
[10] Roel Aaij et al. (LHCb Collaboration), Measurement of the ratio of branching fractions $\mathscr{B}\left(B \rightarrow D^{*} \tau v\right) / \mathscr{B}\left(B \rightarrow D^{*} \mu v\right)$, Phys. Rev. Lett. 115111803 (2015) [Addendum: Phys. Rev. Lett. 115, no.15, 1420159901 (2015)] [arXiv:1506.08614].

[11] S. Hirose et al. (Belle Collaboration), Measurement of the $\tau$ Lepton Polarization and $R\left(D^{*}\right)$ in the Decay $B \rightarrow D^{*} \tau v_{\tau}$, Phys. Rev. Lett. 118211801 (2017) [arXiv:1612.00529].

[12] J. P. Lees et al. (BaBar Collaboration), Branching fraction and form-factor shape measurements of exclusive charmless semileptonic B decays and determination of $\left|V_{u b}\right|$, Phys. Rev. D 86092004 (2012) [arXiv:1208.1253].

[13] H. Ha et al. (Belle Collaboration), Measurement of the decay $B^{0} \rightarrow \pi^{-} \ell^{+} v$ and determination of $\mid V_{u b}$, Phys. Rev. D 83, 071101 (2011) [arXiv:1012.0090].

[14] J.P. Lees et al. (BaBar Collaboration), Study of $B \rightarrow X_{u} \ell v$ decays in B B events tagged by a fully reconstructed B-meson decay and determination of $\left|V_{u b}\right|$, Phys. Rev. D 86032004 (2012) [arXiv:1112.0702]

[15] P. Urquijo et al. (Belle Collaboration), Measurement Of $\left|V_{u b}\right|$ From Inclusive Charmless Semileptonic B Decays, Phys. Rev. Lett. 104021801 (2010) [arXiv:0907.0379]. 